\title{
Safety of switching from brand-name to generic levetiracetam in patients with epilepsy
}

\author{
This article was published in the following Dove Press journal: \\ Drug Design, Development and Therapy \\ 3 August 2017 \\ Number of times this article has been viewed
}

\section{Magdalena Bosak \\ Agnieszka Słowik \\ Wojciech Turaj}

Department of Neurology, Jagiellonian University Medical College, Kraków, Poland
Correspondence: Magdalena Bosak Department of Neurology, Jagiellonian University Medical College, Botaniczna St. 3, 3I-503 Kraków, Poland $\mathrm{Tel}+48$ I2 4248600

Fax +48 I2 4248626

Email magdalena.bosak@uj.edu.pl
Purpose: The approach to the use of generic antiepileptic drugs has recently evolved from major concern to general acceptance, but the evidence related specifically to the safety of switching from brand-name to generic levetiracetam (LEV) is scarce. The aim of the study was to assess the risk of increased frequency of seizures or other adverse events after replacement of a brand-name LEV with a generic one.

Patients and methods: This retrospective analysis included 159 patients treated with LEV in a tertiary outpatient epilepsy clinic. We included all patients diagnosed with epilepsy who were treated with LEV as at March 1, 2013. Most patients were forced to switch to the generic LEV because of the sudden rise in cost of the branded LEV. We recorded data on age, sex, age at onset of epilepsy, type of epilepsy, and its treatment. We analyzed data from one visit before potential switching and from two visits after the potential switching. The interval between visits was typically 3 months. We registered an increase in the frequency of seizures and in the occurrence of adverse events.

Results: Among 151 subjects who switched to generic LEV after March 1, 2013, increased frequency of seizures was noted in 9 patients $(6 \%)$ during the first follow-up visit. Patients with increased frequency of seizures did not differ from other patients regarding sex, age, age at the onset of epilepsy, and the median dose of LEV before switching or the median duration of treatment with LEV before switching. Two patients returned to brand-name LEV. Adverse events were noted in six other patients (4\%) and included somnolence, irritability, or dizziness.

Conclusion: Switching from brand-name to generic LEV is generally safe.

Keywords: levetiracetam, generic, branded, epilepsy, switching, adverse event

\section{Introduction}

At present, generic formulations of all conventional and most of the newer antiepileptic drugs (AEDs) are available. The use of generic AEDs substantially reduces the costs of treatment and is therefore encouraged by regulatory bodies (such as US Food and Drug Administration [FDA]) and health insurance systems. On the other hand, switching from brand-name to generic formulations has raised concerns among physicians and patients regarding loss of seizure control and occurrence of adverse events.

The approach to the use of generic AEDs has changed over the recent years. Initially, most professional societies, including American Academy of Neurology, ${ }^{1}$ Italian League Against Epilepsy, ${ }^{2}$ American Epilepsy Society, ${ }^{3}$ and National Institute for Health and Clinical Excellence ${ }^{4}$ issued statements against compulsory switching that were based mostly on the results of observational studies and case series. Over the years, a growing body of evidence, including a very recent randomized study, 5 suggested reasonable safety of the switching and led to the reversal of the opinions and statements released recently by professional bodies. In 2016, the American Epilepsy 
Society acknowledged that drug formulation substitution with FDA-approved generic products reduces cost without compromising efficacy. ${ }^{6}$

A majority of studies that analyzed switch from the brand-name to generic AEDs included patients taking various AEDs $^{7-11}$ or lamotrigine. ${ }^{12}$ The risk related specifically to the switching from branded to generic levetiracetam (LEV) was assessed only in two studies with discordant results. ${ }^{13,14}$ Thus, the evidence is scarce and justifies further evaluation.

The pharmaceutical policy in Poland includes reimbursement of some medications, including AEDs. Every 2 months, a Ministry of Health issues the list of the medications (brand-name and generic ones) that are refunded either by specific proportion (eg, 50\%) or to the specified price. On March 1, 2013, the price of the brand-name LEV increased by about 20-30 times (depending on the formulation) and most patients were forced to switch to generic formulations because of financial issues (the price of the generic LEV remained at the same level). Therefore, we planned to analyze the consequences of such switching among epileptic patients treated in the tertiary epilepsy outpatient clinic. Our aim was to assess the risk of increased frequency of seizures or other adverse events after replacement of the branded LEV with the generic one.

\section{Patients and methods}

\section{Patients}

This study was a retrospective analysis of patients who were treated with LEV in the tertiary outpatient epilepsy clinic at the Department of Neurology, Hospital University of Krakow, Poland. We included all patients diagnosed with epilepsy who were treated with LEV on March 1, 2013. This date was a turning point in the management of those patients because a vast majority of them were expected to switch from the brand-name to the generic LEV due to a substantial increase in the price of brand-name LEV (see Introduction).

The protocol of the study followed the principles included in the Declaration of Helsinki and was approved by the Bioethical Committee of the Jagiellonian University of Krakow. Patients' consent to review their medical records was not required by the committee because the only person allowed to do so (M.B.) was the physician who took care of all those patients. Two other authors who analyzed and interpreted data used the electronic database without details that would enable patient identification.

\section{Methods}

Using the electronic database, we recorded data on age, sex, age at onset of epilepsy, and treatment of epilepsy (mono- or polytherapy, AEDs used, and the dose and formulation of LEV). The type of epilepsy was retrospectively classified with the terminology used in a recent International League Against Epilepsy position paper on the classification of epilepsies (generalized, focal, combined generalized, and focal or unknown) to facilitate future comparisons. ${ }^{15} \mathrm{We}$ analyzed data from one visit before potential switching and from two visits after the potential switching. The interval between visits was typically 3 months. We noted the baseline frequency of seizures and its potential change after switching. We also registered potential adverse events.

Qualitative variables were characterized with numbers and percentages. Quantitative variables were described with the use of a median with interquartile range (IQR) due to the skewed distribution. Chi-square test (or Fisher exact test, where appropriate) was used to assess the significance of the differences between the qualitative data. Mann-Whitney $U$-test was used to assess differences between quantitative variables. A $P$-value of $<0.05$ was considered statistically significant. All the analyses were performed using Statistica v. 12.5 (StatSoft Inc., Tulsa, OK, USA).

\section{Results}

Table 1 provides the clinical characteristics of 159 patients who were treated with LEV on March 1, 2013. After this date, 151 subjects switched to the generic LEV within about 1 month (when they ran out of the brand-name medication). Their clinical characteristics are summarized in Table 2. One hundred and nine patients $(72.2 \%)$ received generic LEV produced by TEVA (Levetiracetam TEVA) and 42 others $(27.8 \%)$ used generic LEV produced by Adamed (Vetira).

Table I Clinical characteristics of studied patients treated with levetiracetam

\begin{tabular}{ll}
\hline Variable & $\mathbf{N}=\mathbf{I 5 9}^{\mathbf{a}}$ \\
\hline Age (years); median (IQR) & $34(27-42)$ \\
Age at onset of epilepsy (years); median (IQR) & $14(6-20)$ \\
Sex (women) & $91(57.2 \%)$ \\
Type of epilepsy & \\
$\quad$ Generalized & $19(12.0 \%)$ \\
Focal & $132(83.0 \%)$ \\
$\quad$ Unknown & $8(5.0 \%)$ \\
Daily dose of LEV (mg); median (IQR) & $2,000(1,250-3,000)$ \\
Number of AEDs used & \\
I & $15(9.4 \%)$ \\
2 & $90(56.7 \%)$ \\
3 & $50(31.4 \%)$ \\
$\quad$ Unknown & $4(2.5 \%)$ \\
Duration of treatment with LEV (years); & $3(2-4)$ \\
median (IQR) & \\
\hline
\end{tabular}

Note: a One patient used LEV in solution, the others used tablets.

Abbreviations: AED, antiepileptic drug; IQR, interquartile range; LEV, levetiracetam. 
Table 2 Comparison of clinical characteristics of patients treated with the brand-name LEV who were switched to generic LEV or continued treatment with the brand-name LEV

\begin{tabular}{lll}
\hline Variable & $\begin{array}{l}\text { Patients who switched } \\
\text { to generic LEV (n= I I I) }\end{array}$ & $\begin{array}{l}\text { Patients who continued treatment } \\
\text { with the brand-name LEV (n=8) }\end{array}$ \\
\hline Age (years); median (IQR) & $34(28-42)$ & $30.5(20.5-44.5)$ \\
Age at onset of epilepsy (years); median (IQR) & $14(6-20)$ & $8.5(3-15.5)$ \\
Sex (women) & $88(58.3 \%)$ & $3 / 8$ \\
Type of epilepsy & & 0 \\
$\quad$ Generalized & $19(12.6 \%)$ & $6 / 8$ \\
Focal & $125(82.8 \%)$ & $2 / 8$ \\
Unknown & $7(4.6 \%)$ & $5(4-5)^{\mathrm{a}}$ \\
Duration of treatment with LEV (years); median (IQR) & $3(2-4)$ & $2,500(\mathrm{I}, 250-3,000)$ \\
Daily dose of LEV (mg); median (IQR) & $2,000(1,000-3,000)$ & 0 \\
Increased frequency of seizures & $9(6.0 \%)$ & 0 \\
Adverse reactions & $6(4.0 \%)$ & \\
Number of AEDs used & & 0 \\
I & $15(9.9 \%)$ & $5 / 8$ \\
2 & $85(56.3 \%)$ & $2 / 8$ \\
3 & $49(32.5 \%)$ & $1 / 8$ \\
NA & $2(1.3 \%)$ & \\
\hline
\end{tabular}

Notes: $\mathrm{a}<0.0 \mathrm{I}$ for the difference in duration of treatment with LEV; other differences were not significant.

Abbreviations: AED, antiepileptic drug; IQR, interquartile range; LEV, levetiracetam; NA, not available.

After switching, patients used the same formulation of LEV (tablets), the same dose per tablet, and the same daily dose.

Other AEDs most commonly used by the patients treated with LEV and one another medication included valproic acid (30 patients), carbamazepine (21 patients), lamotrigine (13 patients), and oxcarbazepine (13 patients). Patients treated with three AEDs most commonly used a combination of LEV with valproic acid and carbamazepine (11 patients), valproic acid and topiramate ( 9 patients), valproic acid and lamotrigine (7 patients), or carbamazepine and lamotrigine (4 patients).

The clinical characteristics of eight other patients who continued treatment with the brand-name LEV is shown in Table 2 . The only variable that differed significantly between those patients and those who switched to generic LEV was the longer duration of treatment with LEV among patients who did not switch.

The increased frequency of seizures $(>30 \%$ of the baseline frequency) was noted in nine patients $(6 \%)$ during the first follow-up visit after switching. In seven patients, the dose of the generic LEV was subsequently increased by $25 \%$ with the resulting decrease of seizure frequency. In two other patients, it was necessary to return to the brand-name LEV, and the frequency of seizures returned to the baseline in both of them.

The subgroup of nine patients with increased frequency of seizures comprised four women and five men; all patients had focal epilepsy and all received more than one AED for their epilepsy (seven patients took two AEDs and two patients were treated with three AEDs). Medications used in polytherapy of epilepsy in those subjects included oxcarbazepine (2 patients), carbamazepine (2), valproic acid (1), gabapentin (1), lamotrigine (1), lamotrigine and valproic acid (1), and clobazam and valproic acid (1). Patients in this subgroup reported no adverse events other than increased frequency of seizures.

The adverse events were noted in six other patients (4.0\%) at the first follow-up visit after switching and included somnolence ( 2 patients), irritability (2), or somnolence and dizziness (2). All adverse events disappeared at the second follow-up visit and did not lead to the modification of treatment.

The group of patients with increased frequency of seizures did not differ from patients with unchanged frequency of seizures in terms of age (36 [34-51] vs 33 [28-42] years, respectively; $P=0.15)$, age at the onset of epilepsy (16 [5-43] vs 14 [6-20] years, respectively; $P=0.55$ ), median dose of LEV before switching $(2,000[1,500-3,000]$ vs 2,000 $[1,000-3,000] \mathrm{mg}$, respectively; $P=0.60)$ and the median duration of treatment with LEV before switching (4 [3-4] vs 3 [2-4] years, respectively; $P=0.17$ ).

We did not observe any change in seizure frequency or additional adverse events in eight patients who continued treatment with the brand-name LEV.

\section{Discussion}

This retrospective analysis of epileptic patients who switched from the brand-name LEV to generic LEV suggests that such 
a change is generally safe. Increased frequency of seizures was noted only in nine patients $(6 \%)$ and only two of them required switchback to the brand-name LEV. Adverse events were noted in $4 \%$ of patients; they were mild, transient, and did not lead to discontinuation or switchback.

Various AEDs may exhibit different effects when switched from the brand-name to generic formulations. Our study is only the third to report findings related specifically to LEV; thus, the evidence is still equivocal. Our findings are at variance with a similar retrospective observation published by Chaluvadi et al. ${ }^{13}$ These authors observed increased frequency of seizures in $19.3 \%$ of patients (three times more often than we did). Also, as much as $42.8 \%$ of their patients were switched back to the brand-name LEV, which is in sharp contrast to two patients who required such a change in our sample. The frequency of adverse events was similar in those two studies (3.3\% vs $4.0 \%$ ).

These discordant results may be partially explained by the different characteristics of populations studied. More than $90 \%$ of our patients had drug-resistant epilepsy and were treated with multiple medications. It stems from the fact that before March 1, 2013 brand-name (and generic) LEV was reimbursed only for patients with drug-resistant epilepsy. Also, the tertiary outpatient clinic had a disproportionately high proportion of patients on polytherapy. The study sample analyzed by Chaluvadi et al comprised $35.5 \%$ of patients on monotherapy. It may be argued that patients on monotherapy are more prone to increased frequency of seizures, as by definition, there is only one medication to control seizures.

On the other hand, our results are consistent with the second and more recent study related to switching from brand-name to generic LEV. ${ }^{14}$ These authors analyzed 59 patients and did not observe increased frequency of seizures after switching from brand-name LEV to generic during a 6-month follow-up. In their study, two patients (3.4\%) restarted the therapy with the branded LEV because of increased frequency of seizures.

Increased frequency of seizures in a small percentage of patients who switched from brand-name to generic LEV can be attributed to several mechanisms.

The early observational studies pointed to the increased frequency of seizures, occurrence of adverse events, and actual increase of costs when brand-name AEDs were switched to the generic ones. ${ }^{7,11,16,17}$ Consequently, some authors argued that the requirements related to the bioequivalence of AEDs were too permissive, especially for medications with narrow therapeutic indices. ${ }^{18}$ Subsequent studies, however, led to an important change in the paradigm regarding the use of generic AEDs. Ting et $\mathrm{al}^{19}$ confirmed bioequivalence of the brand-name and generic lamotrigine; Privitera et $\mathrm{al}^{5}$ showed bioequivalence of the several generic formulations of lamotrigine. Both studies did not reveal any change in frequency of seizures or incidence of adverse events because of the switching between various formulations of lamotrigine. Similar observations were made in population-based studies on lamotrigine ${ }^{13,20}$ or various AEDs. ${ }^{21}$ Finally, Davit et a ${ }^{22}$ reviewed 2070 clinical bioequivalence studies of oral generic drugs approved by the FDA over 12 years and confirmed the validity of the current criteria used for the approval of generic medications. It seems, therefore, unlikely that the differences in bioequivalence might explain the increased frequency of seizures after switching.

Such increased frequency of seizures in some patients can be related to the natural course of epileptic disorder in which the periods of better or worse control of seizure can interlace.

Also, some authors suggest that the refilling process itself may be associated with the increased frequency of seizures irrespective of whether refilling involved brand-name or generic $\mathrm{AED}^{10}$ or different generic AEDs. ${ }^{21}$ The explanations for this phenomenon may include minor but important changes in bioavailability or lapses in pharmacotherapy continuity related to the refilling process.

We believe that some additional psychological aspects associated with the process of switching can contribute to the increased frequency of seizures in a small minority of patients. Previous concerns of prescribing physicians, based on relevant statements and warnings, might result in more detailed explanations and precautions that would in fact make patients less reassured. At present, when the bioequivalence of brand-name and generic AED is supported by robust evidence, physicians and patients are less prone to the stress related to the process of switching, which may act as a selffulfilling prophecy. In our study, all patients were managed by the single physician who provided consistent and identical information on switching to all subjects.

We acknowledge some limitations in our study, mainly its retrospective design and relatively small sample size $(n=159)$. The small number of patients who did not switch and the small number of those who experienced adverse events after switching make meaningful comparisons difficult. On the other hand, two other published studies related specifically to LEV and discussed earlier included 59 and 260 patients. We did not assess the serum levels of LEV before and after switching. At the time of the study, measurement of LEV levels was not available at our laboratory. 


\section{Conclusion}

The switching from brand-name to generic LEV is generally safe. The proportion of patients who experienced increased frequency of seizures was small (6\%), as was the number of patients who returned to the brand-name LEV after a 6-month follow-up (1.3\%).

\section{Disclosure}

Magdalena Bosak received speaking fees and travel bursaries from UCB Pharma, TEVA Pharmaceuticals, and Adamed, and reports no other conflicts of interest in this work. Agnieszka Słowik and Wojciech Turaj report no conflicts of interest in this work.

\section{References}

1. Liow K, Barkley GL, Pollard JR, Harden CL, Bazil CW; American Academy of Neurology. Position statement on the coverage of anticonvulsant drugs for the treatment of epilepsy. Neurology. 2007;68(16):1249-1250.

2. Perucca E, Albani F, Capovilla G, Bernardina BD, Michelucci R, Zaccara G. Recommendations of the Italian League against Epilepsy working group on generic products of antiepileptic drugs. Epilepsia. 2006;47(Suppl 5):16-20.

3. American Epilepsy Society. AES position on the substitution of different formulations of antiepileptic drugs for the treatment of epilepsy; 2007. Available from: https://www.aesnet.org/sites/default/files/file_attach/ AboutAES/PositionStatements/position\%20statement $\% 20$ on $\% 20$ substitution $\% 20 \mathrm{of} \% 20$ generic\%20formulations.pdf. Accessed March 1, 2017.

4. National Institute for Health and Care Excellence (NICE). Epilepsies: diagnosis and treatment. Clinical guideline [CG137]; 2012 [updated 2016]. Available from: http://www.nice.org.uk/guidance/cg137. Accessed January 20, 2017.

5. Privitera MD, Welty TE, Gidal BE, et al. Generic-to-generic lamotrigine switches in people with epilepsy: the randomized controlled EQUIGEN trial. Lancet Neurol. 2016;15(4):365-372.

6. Vossler DG, Anderson GD, Bainbridge J. AES position statement on generic substitution of antiepileptic drugs. Epilepsy Curr. 2016;16(3): 209-211.

7. Andermann F, Duh MS, Gosselin A, Paradis PE. Compulsory generic switching of antiepileptic drugs: high switchback rates to branded compounds compared with other drug classes. Epilepsia. 2007;48(3): 464-469.
8. Rascati KL, Richards KM, Johnsrud MT, Mann TA. Effects of antiepileptic drug substitutions on epileptic events requiring acute care. Pharmacotherapy. 2009;29(7):769-774.

9. Zachry WM 3rd, Doan QD, Clewell JD, Smith BJ. Case-control analysis of ambulance, emergency room, or inpatient hospital events for epilepsy and antiepileptic drug formulation changes. Epilepsia. 2009; 50(3):493-500.

10. Gagne JJ, Avorn J, Shrank WH, Schneeweiss S. Refilling and switching of antiepileptic drugs and seizure-related events. Clin Pharmacol Ther. 2010;88(3):347-353.

11. Labiner DM, Paradis PE, Manjunath R, et al. Generic antiepileptic drugs and associated medical resource utilization in the United States. Neurology. 2010;74(20):1566-1574.

12. Hartung DM, Middleton L, Svoboda L, McGregor JC. Generic substitution of lamotrigine among medicaid patients with diverse indications: a cohort-crossover study. CNS Drugs. 2012;26(8):707-716.

13. Chaluvadi S, Chiang S, Tran L, Goldsmith CE, Friedman DE. Clinical experience with generic levetiracetam in people with epilepsy. Epilepsia. 2011;52(4):810-815.

14. Vari MS, Pinto F, Mencaroni E, et al. Safety of overnight switch from brand-name to generic levetiracetam. Clin Drug Investig. 2016;36(1): 87-91.

15. Scheffer IE, Berkovic S, Capovilla G, et al. ILAE classification of the epilepsies: position paper of the ILAE commission for classification and terminology. Epilepsia. 2017;58(4):512-521.

16. Kesselheim AS, Stedman MR, Bubrick EJ, et al. Seizure outcomes following the use of generic vs brand-name antiepileptic drugs: a systematic review and meta-analysis. Drugs. 2010;70(5):605-621.

17. Duh MS, Paradis PE, Latrémouille-Viau D, et al. The risks and costs of multiple-generic substitution of topiramate. Neurology. 2010;72(24): 2122-2129.

18. Rediguieri CF, Zeredo JL. Assessing bioequivalence of antiepileptic drugs: are the current requirements too permissive? J Pharm Pharm Sci. 2014;17(2):220-228.

19. Ting TY, Jiang W, Lionberger R, et al. Generic lamotrigine vs brandname Lamictal bioequivalence in patients with epilepsy: a field test of the FDA bioequivalence standard. Epilepsia. 2015;56(9):1415-1424.

20. Lessing C, Ashton T, Davis P. The impact on health outcomes and healthcare utilisation of switching to generic medicines consequent to reference pricing: the case of lamotrigine in New Zealand. Appl Health Econ Health Policy. 2014;12(5):537-546.

21. Kesselheim AS, Bykov K, Gagne JJ, Wang SV, Choudhry NK. Switching generic antiepileptic drug manufacturer not linked to seizures: a case-crossover study. Neurology. 2016;87(17):1796-1801.

22. Davit BM, Nwakama PE, Buehler GJ, et al. Comparing generic and innovator drugs: a review of 12 years of bioequivalence data from the United States Food and Drug Administration. Ann Pharmacother. 2009;43(10):1583-1597.

\section{Publish your work in this journal}

Drug Design, Development and Therapy is an international, peerreviewed open-access journal that spans the spectrum of drug design and development through to clinical applications. Clinical outcomes, patient safety, and programs for the development and effective, safe, and sustained use of medicines are the features of the journal, which

\section{Dovepress}

has also been accepted for indexing on PubMed Central. The manuscript management system is completely online and includes a very quick and fair peer-review system, which is all easy to use. Visit http://www.dovepress.com/testimonials.php to read real quotes from published authors. 